\title{
Performance Improvement of Cloud Computing Data Centers Using Energy Efficient Task Scheduling Algorithms
}

\author{
A.S.Umesh ${ }^{1}$, Dr. Parveen Kumar ${ }^{2}$ \\ ${ }^{1}$ Research Scholar, Computer Science \& Engg, N I M S University, Jaipur, Rajastan, India \\ ${ }^{2}$ Professor, Dept. of Computer Science \& Engg., N I M S University, Jaipur, Rajastan, India
}

\begin{abstract}
Cloud computing is a technology that provides a platform for the sharing of resources such as software, infrastructure, application and other information. It brings a revolution in Information Technology industry by offering on-demand of resources. Clouds are basically virtualized datacenters and applications offered as services. Data center hosts hundreds or thousands of servers which comprised of software and hardware to respond the client request. A large amount of energy requires to perform the operation.. Cloud Computing is facing lot of challenges like Security of Data, Consumption of energy, Server Consolidation, etc. The research work focuses on the study of task scheduling management in a cloud environment.

The main goal is to improve the performance (resource utilization and redeem the consumption of energy) in data centers. Energy-efficient scheduling of workloads helps to redeem the consumption of energy in data centers, thus helps in better usage of resource. This is further reducing operational costs and provides benefits to the clients and also to cloud service provider. In this abstract of paper, the task scheduling in data centers have been compared. Cloudsim a toolkit for modeling and simulation of cloud computing environment has been used to implement and demonstrate the experimental results. The results aimed at analyzing the energy consumed in data centers and shows that by having reduce the consumption of energy the cloud productivity can be improved.
\end{abstract}

Keywords - Data Center, Cloud computing, Virtual Machines, Physical Machines, Workloads, Energy, Utilization of Resources, Operating System, etc.

\section{INTRODUCTION}

This constitutes cloud computing, the evolution of cloud computing, other technologies related like grid.

It also discuss characteristics of cloud, cloud computing services. The evolution of the cloud goes phase by phase that include the Grid Computing, Distributed Computing. Cloud computing is used first in 1950s, the time during which large-scale mainframes were available in the business industry. The hardware used by the mainframe was installed in a big room and all users are accessing the mainframe through terminals. Later in the year 1970, the IBM launches OS having a number of virtual machines at a single machine. The Virtual machine Operating System has taken the application of $1950 \mathrm{~s}$, that is of sharing the access to a mainframe to a higher level by considering a number of virtual machines providing different accessible machines at a single physical machines. Idea of cloud computing was first showed by J.C.R Licklider and John McCarthy in 1969. The vision behind this is that everyone goes interconnected and thus able to access data through anywhere.

The data are stored in a data center (a centralized infrastructure), which is a vast data storage space. The processing of the request or data performed through servers thus availability and security of the data will be addressed. The service provider and the clients has an agreement for the usage known as SLA (Service level Agreement). Then in 1999, salesforce.com put this idea to an application. Then in 2002, a Cloud based services of web launched by Amazon. It provides on demand services to the subscribed users. There are many proposed definitions of the Cloud computing due to its growing popularity defining its characteristics. Some of the definitions given by many wellknown scientists and organizations are: Rajkumar Buyya defines the Cloud computing in terms of its utility to end user as A Cloud is a type of parallel and distributed system consisting of a collection of interconnected and virtualized computers that are dynamically provisioned and presented as one or more unified computing resources based on service-level agreements established through negotiation between the service provider and consumers [1]. 
National Institute of Standards and Technology (NIST) defines Cloud computing as follows: Cloud computing is a model for enabling convenient, on-demand network access to a shared pool of configurable computing resources (e.g., networks, servers, storage, applications, and services) that can be rapidly provisioned and released with minimal management effort or service provider interaction. This Cloud model promotes availability and is composed of five essential characteristics, three service models, and four deployment models [2]. Cloud computing also defined as A style of computing where IT-enabled capabilities are delivered as a service to end users using internet.

\section{PROPOSED TECHNIQUE FOR SCHEDULING WORKLOADS}

PaaS Layer includes the Heterogeneous Workload Consolidation technique to calculate the energy consumption of the data center and also gives the information about the SLA violation, as the allocation policies are implemented on PaaS layer which are followed by the IaaS layer.

\section{Design of Solution}

The solution to the problem Energy Efficient Scheduling of Workload has designed by the proposed Technique and DFD. Following section presents the design of the solution of the proposed Technique and Data Flow Diagrams. Figure shows the layered architecture of Cloud computing. PaaS Layer includes the Heterogeneous Workload Consolidation technique to calculate the energy consumption of the data center and also gives the information about the SLA violation, as the allocation policies are implemented on PaaS layer which are followed by the IaaS layer.

\section{Data Flow Diagram}

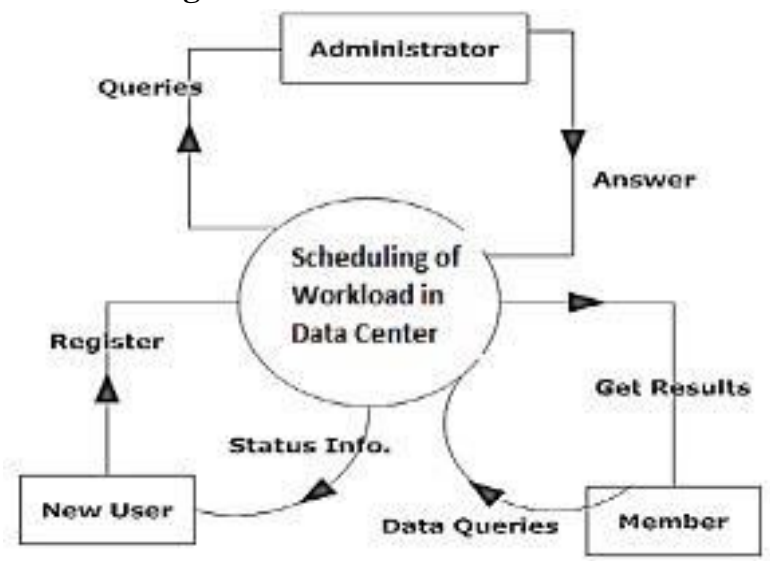

Data Flow Diagrams (DFD) of Heterogeneous Workload Consolidation Technique implemented by designing a Workload Consolidation Cloud Portal (WC Cloud Portal) is shown in Figure, which shows the context level (level0)
DFD of the WC Cloud Portal with three entities- new user (want to register),member (registered user) and administrator.

\section{PROPOSED ALGORITHM}

Below is the proposed algorithm to calculate the power consumption of the the data center .

- Input: Set of tasks and servers Output: Scheduling of tasks to servers

for Each Task x of type i do

- for Each $\mathrm{Sj}$ do

- Calculate server energy consumption $[\mathrm{Ei} ; \mathrm{j}]=\mathrm{Pi} ; \mathrm{j}_{-}$ ti;j

- if $E(i ; j) \_E(a ; b)$ then

- $a=i ; b=j$

end

end

end

while unscheduled tasks remain do

for Each

$\mathrm{Sj}$

do

Calculate energy consumption

Assigned task to e_cient server

end

end

Schedule Task

End

\section{PROPOSED TECHNIQUE WITH BETTER UTILIZATION}

Below is the proposed algorithm to calculate the power consumption of the datacenter by the Workload. The algorithm takes host list as an input and gives VM list of the VMs and power consumed by the data center.

- Input: Set of tasks and servers Output: Scheduling of tasks to servers

- for Each Task x of type i do

- Input the M number of Clouds with L number of Virtual Machines associated with Each cloud.

- Input $\mathrm{N}$ number of user process request with some parameters specifications like arrival time, process time, required memory etc., i.e. host list.

- Arrange the process requests in order of memory requirement

- Power estimatePower(host; V m)

- for Each Task x do

- For each Vm in Vmlist Do 
- if host has enough resourse for Vm Then

- Power estimatePower(host; V m)

- else

- Calculate server energy consumption $[\mathrm{Ei} ; \mathrm{j})=\mathrm{Pi} ; \mathrm{j}_{-}$ ti;j t m:getUtil()hostUtil

- end

- end

- return Vmlist, Energy

- end

\section{TOOLS FOR SETTING CLOUD}

\section{Environment Cloudsim}

CloudSim is an extensible simulation toolkit that enables modeling and simulation of Cloud computing systems and application provisioning environments. The CloudSim toolkit supports both system and behavior modeling of Cloud system components such as data centers, virtual machines and resource provisioning policies. It implements generic application provisioning techniques that can be extended with ease and limited effort.

\section{CLOUDSIM ARCHITECTURE}

Figure shows the multi-layered design of the CloudSim software framework and its architectural components. The CloudSim simulation layer provides support for modeling and simulation of virtualized Cloud-based data center environments including dedicated management interfaces for VMs, memory, storage, and bandwidth.

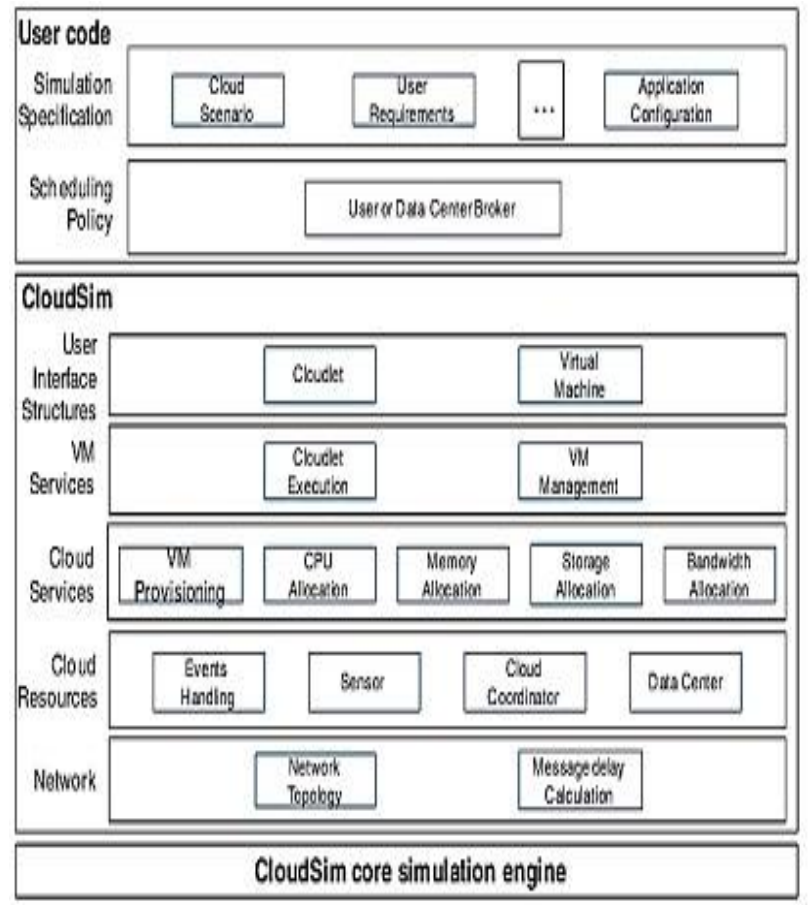

Fig. Cloudsim Simulation

\section{NetBeans}

NetBeans is an integrated development environment for developing primarily with Java, but also with other languages. The NetBeans IDE is written in Java and can run on Windows, OS X, Linux. The NetBeans Platform allows applications to be developed from a set of modular software components called modules. Applications based on the NetBeans Platform including the NetBeans IDE itself can be extended by third party developers.

\section{IMPLEMENTATION OF THE PROPOSED TECHNIQUE}

The technique has been implemented the heterogeneous workload in CloudSim Toolkit by using Netbeans. I have taken a different number of processors, different number of tasks and execution times for each task in each processor as input.

\section{RESULTS}

It can be concluded from the results that the allocation of the virtual machines help to save the energy consumption as the workload will be allocated to the virtual machine having less utilization. So, I can set the threshold utilization of the node according to the variability of the workloads.

\section{CONCLUSION}

This gives the introduction of the Cloud computing technique and discusses various workload allocation techniques to efficient manage workloads. In this work a task assignment technique to manage the energy consumption of the data center has been proposed. Technique has been developed in java, deployed on CloudSim toolkit and Experimental results have been gathered.

\section{REFERENCES}

[1] R. Buyya, C. Yeo, and S. Venugopal, -Marketoriented cloud computing: Vision, hype, and reality for delivering it services as computing utilities, in Proceedings of the 10th IEEE International Conference on High Performance Computing and Communications (HPCC-OB, IEEE CS Press. Los Alamitos, CA. USA), 2008.

[2] P. Mell, and T. Grance, - The NIST Definition of Cloud computing, National Institute of Standards and Technology, 2009.

[3] Rodrigo N. Calheiros, Rajiv Ranjan, Anton Beloglazov, Cesar A. F. De Rose and RajkumarBuyya, - CloudSim: a toolkit for modeling and simulation of cloud computing environments and evaluation of 
resource provisioning algorithms Software: Practice and Experience, Wiley Press, New York, USA, 2010.

[4] S. Garg and R. Buyya, - Green Cloud Computing and Environmental Sustainability, Harnessing Green IT: Principles and Practices, S. Murugesan and G. Gangadharan (eds), Wiley Press, UK, in press, accepted on April 2, 2011.

[5] Growth in data center electricity use 2005 to 2010, Analytics Press, Tech. Rep., 2011.

[6] G. Chen, W. He, J. Liu, S. Nath, L. Rigas, L. Xiao, and F. Zhao.Energy-aware server provisioning and load dispatching for connection-intensive internet services. In Proceedings of the 5th USENIX Symposium on Networked Systems Design and Implementation, NSDI08, pages 337350, Berkeley, CA, USA, 2008. USENIX Association. Conclusion and Future Scope

[7] Aasys, -Virtualization Basics, Vol. 6, Issue 9, September 2008.

[8] L. Minas and B. Ellison, Energy Efficiency for Information Technology: How to Reduce Power Consumption in Servers and Data Centers. Intel Press, 2009.

[9] L. A. Barroso and U. Holzle, The case for energyproportional computing, Computer, vol. 40, no. 12, pp. 3337, 2007.

[10] M. Steinder , D. Carrera, I. Whalley, J. Torres and E. Ayguade, - Managing SLAs of heterogeneous workloads using dynamic application placement, HPDC '08 Proceedings of the 17th international symposium on High performance distributed computing, New York, USA, 2008

[11] Gaweda , M. Steinder, I. Whalley, D. Carrera, and D. M. Chess, -Server virtualization in autonomic management of heterogeneous workloads, Integrated Network Management, page no. 139-148, 2007.

[12] YatendraSahu, R.K. Pateriya, Rajeev Kumar Gupta , - Cloud Server Optimization with Load Balancing and Green Computing Techniques Using Dynamic Compare and Balance Algorithm, Proceedings of 5th International Conference on Computational
Intelligence and Communication Networks, page no. 527- 531,2013.

[13] Ning Liu, Ziqian Dong, Roberto Rojas-Cessa, - Task and Server Assignment for Reduction of Energy Consumption in Datacenters, IEEE 11th International Symposium on Network Computing and Applications ,2012.

[14] Zhibo Wang, Yan-Qing Zhang - Energy-Efficient Task Scheduling Algorithms with Human Intelligence Based Task Shuffling and Task Relocation, IEEE/ACM International Conference on Green Computing and Communications, page no 38-43, 2011.

[15] A. Beloglazov ,R. Buyya, and J. Abawajy, Energyefficient management of data center resources for cloud computing: a vision, architectural elements, 25 Conclusion and Future Scope and open challenges, In: International conference on parallel and distributed processing techniques and applications (PDPTA), Las Vegas, USA, 2010.

[16] P. Ahuja ,A. Verma, and A. Neogi, pMapper: power and migration cost aware application placement in virtualized systems, in Proceedings of the 9th ACM/IFIP/USENIX International Conference on Middleware. Springer-Verlag New York, page no. 243264, 2008.

[17] T. Dillon, C. Wu, and E. Chang, Cloud Computing: Issues and Challenges, in 24th IEEE International Conference on Advanced Information Networking and Applications, page no. 27-33, 2010.

[18] J. Hamilton, Cooperative expendable micro-slice servers (CEMS): low cost, low power servers for Internet-scale services, In the Proceeding of CIDR, 2009.

[19] Manishaben, Jaiswal. "Cloud computing and Infrastructure". International Journal of Research and Analytical Reviews, 4(2), 2017, pp. 742-746.

[20] J. Koomey, Estimating total power consumption by servers in the US and the world, Final report, vol. 15, February 2007 